\title{
Diradical character from the local spin analysis
}

\author{
Eloy Ramos-Cordoba, Pedro Salvador \\ Institut de Química Computacional i Catàlisi (IQCC) and Departament de Química, \\ University of Girona, 17071 Girona, Spain \\ e-mail: Pedro.Salvador@udg.edu
}

\begin{abstract}
Diradical species are analyzed on the light of the local spin analysis. The atomic and diatomic contributions to the overall $\left\langle\hat{S}^{2}\right\rangle$ value are used to detect the diradical character of a number of molecular species mostly in their singlet state, for which no spin density exists. A general procedure for the quantification of diradical character for both singlet and triplet states is achieved by using a recently introduced index that measures the deviation of an actual molecule from an ideal system of perfectly localized spin centers. The index is of general applicability and can be easily determined in equal footing from a multireference or an open-shell single-determinant wave function.
\end{abstract}

Keywords: Local spin analysis, diradical character, benzyne isomers, propellane 


\section{Introduction}

Salem ${ }^{1}$ defined diradicals as molecules with two electrons occupying two near degenerate orbitals. Indeed, how close to degeneracy these orbitals are (HOMOLUMO gap) or more generally the singlet-triplet gap is one of the characteristic features of diradical systems. Diradicals are important in chemistry since they emerge as intermediates of many chemical reactions. ${ }^{2}$ Pure, ideal diradicals such as a dissociated $\mathrm{H}_{2}$ singlet can be easily characterized theoretically from different indicators, depending on the nature of the wave function. However, the quantification of the diradical or diradicaloid ${ }^{3}$ character of short-lived singlet diradicals is not so trivial because the formally unpaired electrons do interact to some extent. There is a continuum between the closed-shell spin-paired and the perfectly localized spin-entangled situations, as exemplified by the dissociation curve of singlet $\mathrm{H}_{2}$.

Several indices have been proposed in the literature in order to detect and quantify the diradical character of molecular systems, the simplest probably being the value of $\left\langle\hat{S}^{2}\right\rangle$ of a broken symmetry spin-unrestricted wave function. ${ }^{4,5}$ For a system with an equal mixture of singlet and triplet components one should expect a $\left\langle\hat{S}^{2}\right\rangle$ value close to $1 .^{6}$ Accordingly, Bachler et al. ${ }^{7}$ proposed the following index

$$
n_{\text {rad }}=1-\sqrt{1-\left\langle\hat{S}^{2}\right\rangle_{B S}},
$$

where $\left\langle\hat{S}^{2}\right\rangle_{B S}$ represents a UHF broken-symmetry wave function. An alternative index can be built making explicit use of the occupation numbers of spin-unrestricted natural orbitals (UNOs). In a system with diradical character, a pair of bonding an antibonding orbitals are typically associated with the two radical sites. The closer to 1 the occupation of the antibonding orbital is, the higher the diradical character. Jung and Head-Gordon ${ }^{3}$ and Bachler et al. ${ }^{8}$ used the occupation numbers obtained from perfect-pairing approaches and Lopez et al. used the occupation number computed at the natural orbital functional (NOF) level of theory ${ }^{9}$ to assess the extent of diradical character of different molecules. Rivero et al. also studied the extent

of radical character from the occupation numbers that are close to one from a spin- 
projected Hartree-Fock calculation. ${ }^{10}$ Similarly, Kamada et al. ${ }^{11}$ used the index,

$$
y=\frac{(1-T)^{2}}{1+T^{2}} \quad \text { and } \quad T=\frac{n_{H O M O}-n_{L U M O}}{2}
$$

where $n_{H O M O}$ and $n_{L U M O}$ are the occupations of the bonding and antibonding UNOs. In a purely closed-shell system $n_{H O M O}=2$ and $n_{L U M O}=0$, and hence $y=0$. When the occupations of the two orbitals are equal the system is a pure diradical and $y=1$.

When a multiconfigurational wave function is used the occupation numbers of the orbitals of the radical sites can be replaced by the weights of appropriate configurations of the $\mathrm{CI}$ expansion. In the simplest two-electrons in two-orbitals (the so-called magnetic orbitals) model, the $2 \times 2 \mathrm{CI}$ wave function is build up from a configuration in which the bonding combination of the magnetic orbitals is doubly occupied, and another that includes the double excitation to the antibonding combination of the magnetic orbitals. Bachler et. al. ${ }^{7}$ proposed the following indicator for diradical character

$$
n_{\text {rad }}^{C I}=\sqrt{2}\left|c_{d}\right|
$$

where $c_{d}$ is the weight of the doubly-excited configuration. Later on, other authors suggested an improved version ${ }^{12}$ that also incorporates the weight of the other configuration

$$
d=2 \sqrt{\frac{c_{0}^{2} c_{d}^{2}}{c_{0}^{2}+c_{d}^{2}}} .
$$

None of the indices described above is of general applicability. Beyond diradicals a signature of polyradical character may be derived from the shape and occupation of the natural orbitals. ${ }^{10,13}$ The applicability of the indices given in Eqns. (1) to (4) is thus restricted to diradical systems that can be well described with a twoelectron two-orbital model. A noteworthy alternative is the analysis of the so-called density of effectively unpaired electrons, $u(\vec{r})$, defined by Takatsuka et al. ${ }^{14}$ as

$$
u(\vec{r})=2 \rho(\vec{r})-\int \rho\left(\vec{r} ; \vec{r}^{\prime}\right) \rho\left(\vec{r}^{\prime} ; \vec{r}\right) d \vec{r}^{\prime}
$$

This quantity can be easily obtained at any level of theory from the first-order density matrix, $\rho\left(\vec{r} ; \vec{r}^{\prime}\right)$, and provides a spatial distribution of the unpaired or "odd" 
electrons in the system, even if the spin density vanishes (e. g. for multiconfigurational singlet wave functions). The total number of unpaired electrons, $N_{D}$, can be recovered upon integration of $u(\vec{r})$ over the whole space. The topology $u(\vec{r})$ and the $N_{D}$ values have been used by Staroverov and Davidson to analyze the evolution of the radical character upon a chemical reaction, e.g. the Cope rearrangement. ${ }^{15,16}$ Cheng and $\mathrm{Hu}^{17}$ found a good correlation between $N_{D}$ and the singlet-triplet gap for a set of $\mathrm{B}_{2} \mathrm{P}_{2}$ ring derivative diradicaloids. Moreover, population analysis techniques such as Mulliken ${ }^{16}$ or QTAIM ${ }^{18}$ have also been applied to recover the average number of unpaired electrons on a given atom/fragment. It is worth to note that Mulliken populations of $u(\vec{r})$ are identical to Mayer's free valence index ${ }^{19,20}$ for singlet wave functions.

In singlet diradicals the presence of some local spin associated to a given atom or fragment of the molecular system is assumed. The spin properties of molecular systems are usually characterized by the analysis of the spin density. In fact, spin-unrestricted single-determinant calculations often result in broken-symmetry solutions with non-vanishing spin density. In this case, however, the state of the system is not described as a pure singlet, as it appears contaminated with higher spin states. When a proper multireference wave function is used to describe a pure singlet the spin density exactly vanishes at all points of the space. Yet, one can still invoke the concept of local spin in the system.

Local spins can be retrieved from wave function analysis by a number of decomposition schemes. ${ }^{21-27}$ The most appropriate approach to the problem, as pointed out by Mayer, ${ }^{23}$ is probably the exact decomposition of the expectation value of the spin-squared operator into a sum of atomic and diatomic contributions as

$$
\left\langle\widehat{S}^{2}\right\rangle=\sum_{A}\left\langle\widehat{S}^{2}\right\rangle_{A}+\sum_{A, B \neq A}\left\langle\widehat{S}^{2}\right\rangle_{A B}
$$

A proper formulation of eqn. (6) can provide vanishing one- and two-center terms for restricted single-determinant wave functions (thereby distinguishing electron pairing in bonds from antiferromagnetic coupling), and non-zero ones for pure singlets described by correlated wave functions, thus overcoming the limitation of use of the spin density. The actual expressions for the one- and two-center contributions fulfilling these conditions, henceforth local spin analysis, can be found elsewhere. ${ }^{27}$ 
In the local spin analysis, the $\left\langle\hat{S}^{2}\right\rangle_{A}$ values indicate and quantify the presence of local spin within the molecule, namely on atom/fragment $A$. The magnitude and sign of the diatomic contributions $\left\langle\hat{S}^{2}\right\rangle_{A B}$ with $B \neq A$ inform about the nature of the couplings between these local spins. ${ }^{21,28,29}$ The physical interpretation of the $\left\langle\hat{S}^{2}\right\rangle_{A}$ and particularly $\left\langle\hat{S}^{2}\right\rangle_{A B}$ values is somewhat intricate, and has been recently discussed in detail in several papers. ${ }^{30,31}$

The ability of both local spin methods and the density of effectively unpaired electrons to capture the diradical nature of molecular systems has already been discussed in the recent literature. ${ }^{15,16,32-36}$ However, their use as a general index for the quantification of the diradical character has not yet been fully explored. This is the main goal of the present work.

\section{Computational Details}

Since nondynamical correlation is essential to describe the low-spin components of diradicals, the use of a multireference method is mandatory. All wave functions for the molecular systems studied have been obtained at the CASSCF level with the cc-pVTZ basis set, unless otherwise indicated. For the simple diradical model systems the STO-3G basis set in combination with CASSCF or UHF levels of theory has been used instead. The first- and second-order density matrices have been obtained using a modified version of Gaussian $03^{37}$ and an auxiliary program ${ }^{38}$ that reads and processes the CASSCF outputs. All local spin components are given in atomic units. All calculations have been carried out at the geometrical structure of the molecules optimized at the current level of theory, unless otherwise indicated. The local spin analysis has been performed with the program APOST-3D. ${ }^{39}$ For this work we have make use of the atomic domains provided by the recently introduced topological fuzzy Voronoi cells (TFVC) scheme. ${ }^{40}$ It is a fuzzy-atom based alternative $^{41}$ to Bader's QTAIM domains that produces very similar results with much less computational effort. ${ }^{40}$ 


\section{Results and discussion}

\section{Local spin vs density of effectively unpaired electrons}

In Figures 1 and 2 we plot the evolution of the indices of Eqn. 1-4 for diradical character for a simple model system, namely the dissociation of a singlet $\mathrm{H}_{2}$ into two doublet $\mathrm{H}$ atoms described with minimal basis at the UHF and FCI levels of theory, respectively. The values of the number of effectively unpaired electrons averaged over one of the $\mathrm{H}$ atom, $N_{D}^{H}$ and the local spin, $\left\langle\hat{S}^{2}\right\rangle_{H}$, are also included. The later has been rescaled to vary from 0 to 1 for better comparison.

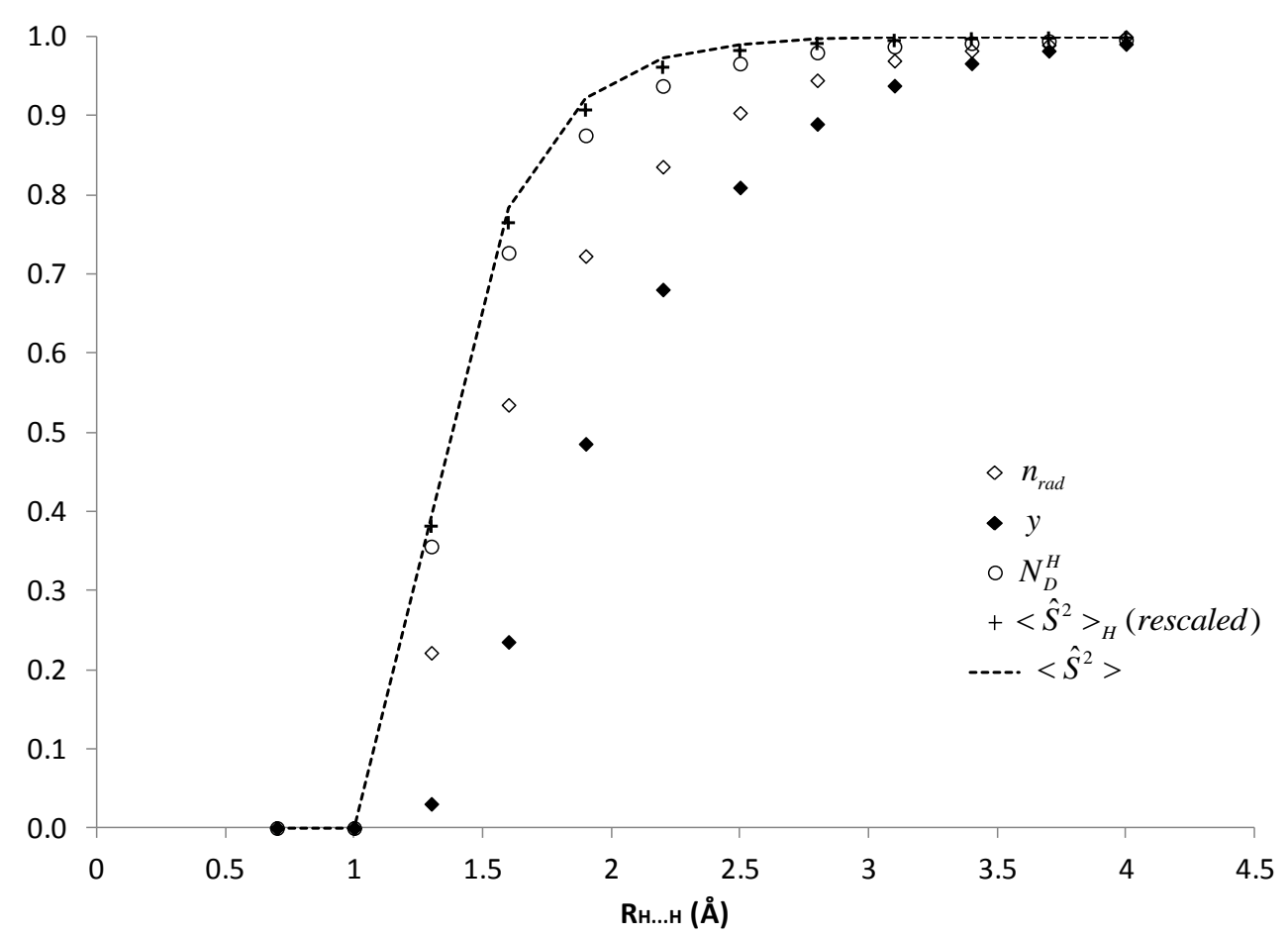

Figure 1: Indices for diradical character along dissociation of $\mathrm{H}_{2}$ model system at the UHF level of theory. $\left\langle\hat{S}^{2}\right\rangle_{H}$ values have been rescaled (see text).

For a single-determinant wave function the diradical character is exactly zero for 
all indices when no BS solution exists (see Figure 1). As the H-H distance stretches and a BS solution is found, the diradical character monotonically increases in all cases. At large distances all indices tend to 1, indicating a perfect diradical. For intermediate distances, the index $y$ from Eqn.(2) seems to underestimate the extent of diradical character with respect to the other indicators. Both the local spin and the number of effectively unpaired electrons closely follow the value of $\left\langle\hat{S}^{2}\right\rangle$. For this model system the $n_{\text {rad }}$ index is equivalent to the occupation of the LUMO orbital $\left(n_{L U M O}\right)$.

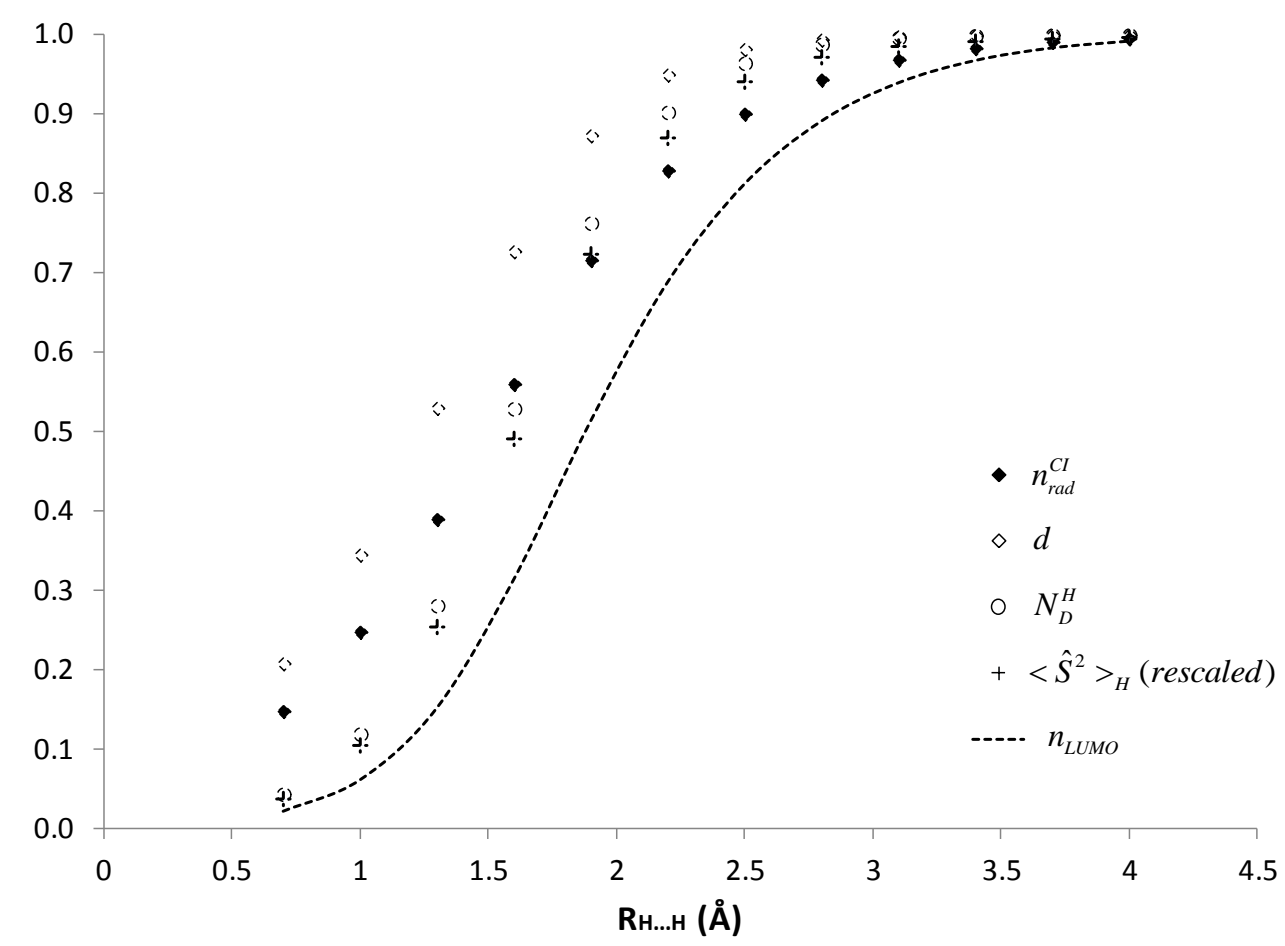

Figure 2: Indices for diradical character along dissociation of $\mathrm{H}_{2}$ model system at the FCI level of theory. $\left\langle\hat{S}^{2}\right\rangle_{H}$ values have been rescaled

For correlated wave functions the diradical character predicted by the different indices is always different from zero. The $d$ index of Eqn. (4) yields a $20 \%$ of 
diradical character for $\mathrm{H}_{2}$ at the equilibrium distance $(0.74 \AA)$, which is probably somewhat too large. The local spin and number of effectively unpaired electrons on the $\mathrm{H}$ atoms give a similar and much smaller diradical character, and the index given in eqn. (3) lies in between. In this case, the occupation of the LUMO (antibonding) orbital consistently yields a smaller diradical character than the other indices. At intermediate atomic distances the differences between all indices are smaller than in the case of the UHF-BS description.

It is worth to note that Clark and Davidson ${ }^{32}$ also applied their local spin formalism to the dissociation profile of diatomics such as $\mathrm{H}_{2}$ at RHF, UHF and FCI levels of theory. In their method, the $\left\langle\hat{S}^{2}\right\rangle_{A}$ value also tends to $3 / 4$ at the dissociation limit, but for interatomic distances near equilibrium it tends to $3 / 8$ of the bond order. The non-vanishing local spin contributions for a genuinely diamagnetic system like $\mathrm{H}_{2}$ at equilibrium distance difficult their use as indicators of diradical character.

We have just seen that both the number of effectively unpaired electrons and the local spin analysis quantify in a similar manner the diradical character for a simple model system. Indeed, several studies have shown that both are very useful tools for the characterization of the spin distribution in actual molecular systems, in particular for singlet states. ${ }^{15,16,32-35}$ However, $u(\vec{r})$ also exhibits some unattractive peculiarities. First of all, the upper bound for $N_{D}$ was found to be $2 N$, where $N$ is the total number of electrons. Thus, the number of effectively unpaired electrons may be larger than the actual number of electrons. ${ }^{42}$ This unphysical upper bound hinders the use of $N_{D}$ as an absolute index for radical character. Another rather puzzling result was found in the dissociation of $\mathrm{O}_{2}$ in its ${ }^{3} \Sigma_{g}^{-}$ground state into two triplet ${ }^{3} P$ oxygen atoms. Staroverov and Davidson ${ }^{42}$ obtained a value of $N_{D}=5$ at the dissociation limit, i.e., each $\mathrm{O}$ atom carries an average of 2.5 unpaired electrons, instead of the expected value of 2 for an isolated triplet. It is worth mentioning that this finding motivated an alternative definition of $u(\vec{r})$ by Head-Gordon, ${ }^{43}$ although not without controversy. ${ }^{44,45}$

We have further explored this paradigmatic system by considering for a number of different electronic states the dissociation of $\mathrm{O}_{2}$ into two $\mathrm{O}$ atoms. In the dissociation limit one can have either two radical centers with two unpaired electrons each (when the $\mathrm{O}_{2}$ dissociates into two triplet ${ }^{3} \mathrm{P}$ oxygen atoms), or no spin centers at all when it dissociates into two ${ }^{1} D$ singlet $\mathrm{O}$ atoms. Note that neither situations can be 
described with eqns. (1) to (4). In Table 1 we collect the values of $N_{D}$ and local spin on the $\mathrm{O}$ atoms upon dissociation for several molecular (and atomic) electronic states. The wave functions have been obtained at the $\operatorname{CASSCF}(8,6) / 6-31 \mathrm{G}^{*}$ level of theory.

Table 1: Number of effectively unpaired electrons $\left(N_{D}\right)$ and local spin values for the $\mathrm{O}$ atoms, $\left\langle\hat{S}^{2}\right\rangle_{\mathrm{O}}$, at the dissociation limit of several $\mathrm{O}_{2}$ molecular electronic states.

\begin{tabular}{cccc}
\hline $\begin{array}{c}\text { Molecular } \\
\text { elec. state }\end{array}$ & $N_{D}$ & $\left\langle\hat{S}^{2}\right\rangle_{\mathrm{O}_{1}} /\left\langle\hat{S}^{2}\right\rangle_{\mathrm{O}_{2}}$ & $\begin{array}{c}\text { Atomic } \\
\text { elec. state }^{a}\end{array}$ \\
\hline${ }^{3} \Sigma_{g}^{-}$ & 5 & $2 / 2$ & ${ }^{3} P /{ }^{3} P$ \\
${ }^{1} \Delta_{g}$ & 4 & $2 / 2$ & ${ }^{3} P /{ }^{3} P$ \\
${ }^{1} \Sigma_{g}^{+}$ & 5 & $2 / 2$ & ${ }^{3} P /{ }^{3} P$ \\
${ }^{1} \Sigma_{u}^{-}$ & 5 & $2 / 2$ & $3{ }^{3} P /{ }^{3} P$ \\
${ }^{3} \Pi_{u}$ & 4 & $2 / 2$ & ${ }^{3} P /{ }^{3} P$ \\
${ }^{1} \Pi_{g}$ & 5 & $2 / 2$ & ${ }^{3} P /{ }^{3} P$ \\
${ }^{1} \Pi_{u}$ & 5 & $2 / 2$ & ${ }^{3} P /{ }^{3} P$ \\
${ }^{1} \Delta_{u}$ & 5.33 & $0 / 0$ & ${ }^{1} D /{ }^{1} D$ \\
${ }^{1} \Pi_{u}$ & 4.99 & $0 / 0$ & ${ }^{1} D /{ }^{1} D$ \\
\hline
\end{tabular}

${ }^{a}$ Atomic electronic states at the dissociation limit

For the ground ${ }^{3} \Sigma_{g}^{-}$state, a value of $N_{D}=5$ is obtained upon dissociation into two triplet ${ }^{3} P$ oxygen atoms, as already noted by Staroverov and Davidson. ${ }^{42}$ However, this is not always the case. For instance, for the dissociation of the ${ }^{1} \Delta_{g}$ and ${ }^{3} \Pi_{u}$ states into two triplet $\mathrm{O}$ atoms, the expected $N_{D}=4$ value is recovered. The ${ }^{1} \Delta_{u}$ state dissociates into two ${ }^{1} D$ singlet oxygen atoms, but the $N_{D}$ value is $16 / 3$, consistent with the uniform distribution of 8 electrons into 6 degenerate $\mathrm{p}$ orbitals. Thus, by looking at the $N_{D}$ values at the dissociation limit one can not distinguish two triplet from two singlet oxygen atoms (in this case the distinction is evident from the energy values). Moreover, different $N_{D}$ values can be obtained for a system consisting of two dissociated triplet oxygen atoms, depending on the overall electronic state. It is worth to note that using Head-Gordon's ${ }^{43}$ alternative formulation one would obtain $N_{D}=4$ in all cases (in fact, as long as the natural occupations are greater or equal than 1).

On the other hand, the local spin values always yield the expected values for the dissociating oxygen atoms. Matito and Mayer ${ }^{24}$ already reported proper asymp- 
totics of the atomic local spin contributions for the lowest-lying triplet and singlet states. We have considered here the dissociation of five more molecular singlet and triplet states that dissociate into two ${ }^{3} P$ oxygen atoms and in all cases $\left\langle\hat{S}^{2}\right\rangle_{\mathrm{O}}=2$ (see Table 1). For the states that dissociate into two singlet ${ }^{1} D$ oxygen atoms, namely ${ }^{1} \Delta_{u}$ and ${ }^{1} \Pi_{u}$, the local spin analysis yields $\left\langle\hat{S}^{2}\right\rangle_{\mathrm{O}}=0$. The diatomic spin components also differentiate when the two oxygen triplets are coupled as a singlet, like in

the ${ }^{1} \Delta_{g}$ state for which $\left\langle\hat{S}^{2}\right\rangle_{\mathrm{O}, \mathrm{O}}=-2$, or as a triplet, like in the ${ }^{3} \Sigma_{g}^{-}$state, for which $\left\langle\hat{S}^{2}\right\rangle_{\mathrm{O}, \mathrm{O}}=-1$ is obtained.

Thus, the local spin analysis appears to be more suitable tool than the number of effectively unpaired electrons when it comes to the formal breaking of more than one bond. This is in essence because the $\left\langle\hat{S}^{2}\right\rangle_{A}$ terms include contributions from the cumulant of the second order-density matrix, whereas the number of effectively unpaired electrons is obtained only from the first-order density matrix. Accordingly, our goal, which is the quantification of diradical character, will be better accomplished by making use of the descriptors obtained from the local spin analysis.

\section{Quantification of diradical character in molecules}

The spin distribution of diradical species has already been analyzed in the light of the number of effectively unpaired electrons and different local spin indicators. Typically studied examples are benzyne isomers. ${ }^{26,46-48}$ Clark and Davidson analyzed their electronic structure making use of the density of effectively unpaired electrons ${ }^{49}$ and also their local spin formalism. ${ }^{32,33}$ The evolution of local spins ${ }^{32}$ and the number of unpaired electrons ${ }^{34}$ along reactive processes involving benzyne were also discussed in detail.

For the present work we have studied a number of diradical and diradicaloid species at equilibrium geometries. The species considered are depicted in Figure 3. For all of them we have performed the local spin analysis, but the results will not be discussed in detail here (for that we refer to the supporting information). Instead, we will focus essentially on the actual quantification of the diradical character. For this purpose, only the atomic contributions of the local spin analysis will be taken into account. 
<smiles>[c]1ccccc1</smiles>

ortho-benzyne

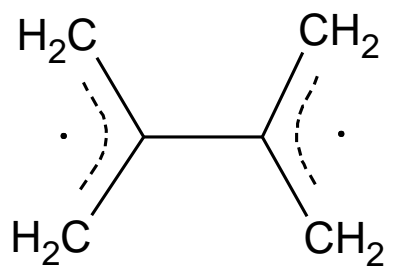

TME

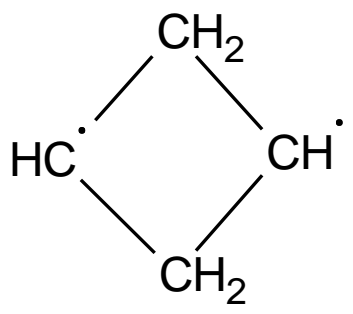

$(\mathrm{CH})_{2}\left(\mathrm{CH}_{2}\right)_{2}$

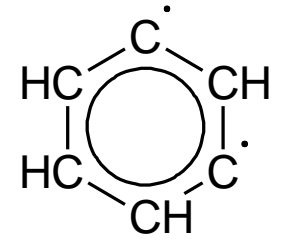

meta-benzyne

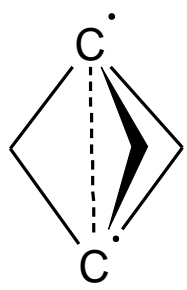

$[1,1,1]$ propellane

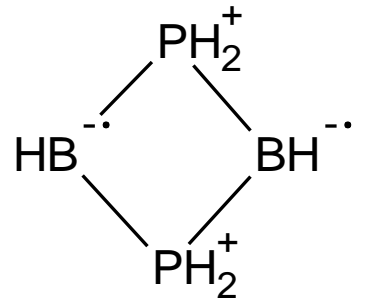

$(\mathrm{BH})_{2}\left(\mathrm{PH}_{2}\right)_{2}$

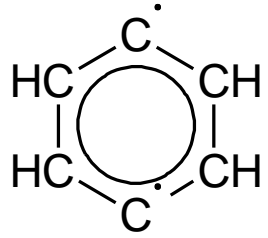

para-benzyne

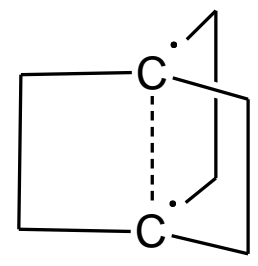

$[2,2,2]$ propellane

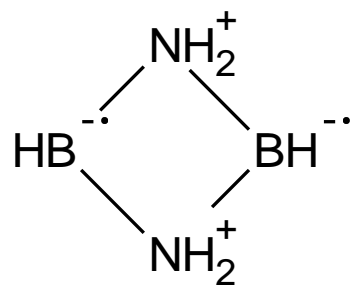

$(\mathrm{BH})_{2}\left(\mathrm{NH}_{2}\right)_{2}$

Figure 3: Diradicals and diradicaloids considered in this work

Most recently we have introduced as a general measure of $k$-radical character the following index

$$
\Delta^{(k)}=\sqrt{\frac{\sum_{A}\left(\left\langle\hat{S}^{2}\right\rangle_{A}-\left\langle\hat{S}^{2}\right\rangle_{A}^{i d}\right)^{2}}{n}},
$$

where the $\left\langle\hat{S}^{2}\right\rangle_{A}^{i d}$ represent the atomic ideal values and $n$ is the total number of 
atoms/fragments considered in the local spin analysis (for most applications the hydrogen atoms can be safely ignored.) ${ }^{31}$ Since the "ideal" value for the diatomic terms depends upon the particular electronic state (singlet, triplet, ... ) and also the type of wave function (broken-symmetry vs multireference), the index only uses the atomic contributions of the local spin analysis. The main advantage of this index is that, contrary to most approaches in the literature, it is calculated in the same manner from both multireference and unrestricted single-determinant wave functions, and for any electronic state. The smaller the $\Delta^{(k)}$ value the closer the system is to a reference picture of $k$ perfectly localized spin centers. So far, the use of eqn. 7 has been restricted to $k=3$, for the quantification of the triradical character. ${ }^{31}$

For diradical or diradicaloid species in singlet-state one can calculate both $\Delta^{(0)}$ and $\Delta^{(2)}$ values. The former will measure average deviation from a nonradical closed-shell picture, and the latter will indicate the deviation from a perfect diradical. This provides a numerical criterion to identify diradicaloids as either diradicals or nonradical species.

Table 2: $k$-radical character index, $\Delta^{(k)}$, for a set of diradicals and diradicaloids. Values in parenthesis computed at the UB3LYP level of theory.

\begin{tabular}{cccc}
\hline & \multicolumn{2}{c}{ Singlet } & Triplet \\
Molecule & $\Delta^{(0)}$ & $\Delta^{(2)}$ & $\Delta^{(2)}$ \\
\hline ortho-benzyne & 0.18 & 0.28 & 0.14 \\
meta-benzyne & 0.28 & 0.19 & 0.13 \\
para-benzyne & 0.49 & 0.12 & 0.12 \\
TME & 0.77 & 0.02 & 0.05 \\
$(\mathrm{CH})_{2}\left(\mathrm{CH}_{2}\right)_{2}$ & $0.41(0.42)$ & $0.12(0.12)$ & $0.12(0.12)$ \\
$(\mathrm{BH})_{2}\left(\mathrm{PH}_{2}\right)_{2}$ & $0.10(0.00)$ & $0.46(0.53)$ & $0.39(0.34)$ \\
$(\mathrm{BH})_{2}\left(\mathrm{NH}_{2}\right)_{2}$ & $0.17(0.18)$ & $0.41(0.40)$ & $0.38(0.36)$ \\
{$[1,1,1]$ propellane } & 0.02 & 0.47 & - \\
{$[2,2,2]$ propellane $\left(\mathrm{R}_{\mathrm{C}-\mathrm{C}}=1.536 \AA\right)$} & 0.01 & 0.36 & - \\
{$[2,2,2]$ propellane $\left(\mathrm{R}_{\mathrm{C}-\mathrm{C}}=1.988 \AA\right)$} & 0.16 & 0.22 & - \\
{$[2,2,2]$ propellane $\left(\mathrm{R}_{\mathrm{C}-\mathrm{C}}=2.532 \AA\right)$} & 0.05 & 0.33 & - \\
\hline
\end{tabular}

The computed $\Delta^{(0)}$ and $\Delta^{(2)}$ values for the species of Figure 3 are gathered on Table 2. The trends of the $\Delta^{(0)}$ and $\Delta^{(2)}$ values along the series of singlet ortho-, 
meta- and para-benzyne are very illustrative. For ortho-benzyne $\Delta^{(0)}=0.18$ and $\Delta^{(2)}=0.28$, indicating a smaller deviation of this species with respect to a closedshell picture. For meta-benzyne the situation is just the opposite, and the system is better identified as a diradical. For para-benzyne the $\Delta^{(2)}$ value is very small $(0.12)$ and much smaller than the $\Delta^{(0)}$ one (0.49), which is consistent with a diradical picture. Thus, both $\Delta^{(0)}$ and $\Delta^{(2)}$ values are able to reproduce the assumed trend ortho $<$ meta $<$ para of the diradical character of benzyne isomers. ${ }^{32,46,47}$ For triplet states only the $\Delta^{(2)}$ values are meaningful. The values are quite small and do not differ too much from one isomer to another. The trend along the series is the same as for the singlet states, i.e., , triplet para-benzyne is more diradical than orthobenzyne.

Sometimes the spin delocalization hinders the recognition of the formal spin centers, like in the well-studied tetramethylenethane (TME) diradical. ${ }^{50,51}$ At the CASSCF level of theory the lowest energy structure has $D_{2}$ symmetry, with a dihedral angle relating the two allyl moieties of $70.6 \mathrm{deg}$. The active space included 6 electrons and 6 orbitals (in the $D_{2 h}$ symmetry they correspond to the set of $6 \pi$ orbitals.) The results of the local spin analysis are discussed in detail in the supporting information. Essentially, the analysis reveals that TME is made up from two independent allyl radicals bonded by the central carbon atoms. The spin distribution among the atoms of the allyl fragments is very similar for both the singlet and triplet states.

Thus, for this molecule it is more appropriate to consider two allyl fragments in eq. 7, instead of all atoms separately. The local spin contribution of a molecular fragment is simply obtained by summing up all atomic and diatomic contributions of the atoms that form the molecular fragment. The $\Delta^{(2)}$ value taking the two allyl moieties as spin centers is very small (0.02) in the singlet state, and somewhat larger in the triplet (0.05) due to the enhanced delocalization of the spins between the two moieties. The $\Delta^{(0)}$ value is clearly too large to consider this system as a closed-shell species at all.

The distinction between a singlet diradical and a nonradical closed-shell species is sometimes not so evident. Diphosphadiboretanes and their analogues are some of the most controversial systems discussed in the literature. Scheschkewitz et al. ${ }^{52}$ 
reported several years ago a 1,3-diphospha-2,4-diboretane derivative singlet diradical that exhibited indefinite stability at room temperature. Several theoretical studies $^{3,8,17,53}$ followed that work, aimed at the quantification of the diradical character of this species and its analogues. We depict on Figure 3 some of these four-member ring diradicaloids. The species $(\mathrm{CH})_{2}\left(\mathrm{CH}_{2}\right)_{2}$ corresponds to a planar transition state structure on the singlet potential energy surface of bicyclobutane (a triplet state lies ca. 2-3 kcal/mol lower in energy.) $(\mathrm{BH})_{2}\left(\mathrm{PH}_{2}\right)_{2}$ is simplest diphosphadiboretane and $(\mathrm{BH})_{2}\left(\mathrm{NH}_{2}\right)_{2}$ is a diaza-analogue of the former. This system is interesting because even though it exhibits a much shorter B-B distance (2.04 $\AA$ ) than in diphosphadiboretane (2.60 ̊), its diradical character was estimated to be smaller. ${ }^{53}$

We have studied these systems with an unrestricted single-determinant wave function (UB3LYP) for both their singlet and triplet states. We have also considered a single-point CASSCF $(2,2)$ wave function at the UB3LYP optimized structures for comparison. For the singlet states, the atomic local spin values are very similar for $\operatorname{CASSCF}(2,2)$ and UB3LYP methods, provided a broken-symmetry solution is found for the latter (the local spin contributions are exactly zero for a restricted single-determinant wave function, as in $\left.(\mathrm{BH})_{2}\left(\mathrm{PH}_{2}\right)_{2}\right)$. Remarkably, a broken-symmetry wave function that yields a wrong value of $\left\langle\hat{S}^{2}\right\rangle$ does seem to provide appropriate atomic $\left\langle\hat{S}^{2}\right\rangle_{A}$ contributions. The flaw of the broken-symmetry solution is found on the diatomic spin-spin interactions between the local spin centers: the UB3LYP values are significantly smaller than the $\operatorname{CASSCF}(2,2)$ ones. Yet, the negative sign still indicates the antiparallel arrangement of the local spins (see supporting information). For triplet states the local spin analysis yields very similar one- and two-center contributions for both methods. This is not surprising since with a $\operatorname{CASSCF}(2,2)$ approach the $m_{S}=|S|$ state is described by a ROHF wave function. Therefore, since the indices of eqn. 7 use only the atomic local spin contributions, their values for a broken-symmetry and a CASSCF wave function will be very similar.

We find that singlet $(\mathrm{CH})_{2}\left(\mathrm{CH}_{2}\right)_{2}$ is best described as a diradical. The $\Delta^{(2)}$ value is similar to that of para-benzyne $(0.12)$, whereas $\Delta^{(0)}$ is much larger. These values are indeed almost the same for $\operatorname{CASSCF}(2,2)$ and $\operatorname{UB} 3 \operatorname{LYP}$ wave functions. The $\Delta^{(0)}$ value for $(\mathrm{BH})_{2}\left(\mathrm{PH}_{2}\right)_{2}$ is trivially zero at the UB3LYP level of theory, as it corresponds to a restricted closed-shell solution. $\operatorname{For} \operatorname{CASSCF}(2,2)$ the value 
slightly increases to 0.10 but still is significantly smaller than the $\Delta^{(2)}$ value. Clearly, this species can not be considered a diradical, in agreement with Jung et al. ${ }^{53}$ For the diaza analogue, $\Delta^{(0)}$ increases to ca. 0.17 and $\Delta^{(2)}$ decreases to 0.41. Thus, $(\mathrm{BH})_{2}\left(\mathrm{NH}_{2}\right)_{2}$ is more diradical than $(\mathrm{BH})_{2}\left(\mathrm{PH}_{2}\right)_{2}$, but still it is best described as a closed-shell species. For triplet states, the local spin analysis reveals in the case of $(\mathrm{BH})_{2}\left(\mathrm{PH}_{2}\right)_{2}$ and $(\mathrm{BH})_{2}\left(\mathrm{NH}_{2}\right)_{2}$ that the four atoms of the ring exhibit similar but small local spin contributions (see supporting information). The large $\Delta^{(2)}$ values for the triplet states of $(\mathrm{BH})_{2}\left(\mathrm{PH}_{2}\right)_{2}$ and $(\mathrm{BH})_{2}\left(\mathrm{NH}_{2}\right)_{2}$ are thus consistent with the observed delocalized-spin picture.

Finally, the nature of the central C-C bond in strained systems such as propellanes has been subjected to debate in the literature for years. The formal picture of these species in the absence of this bond would be a diradical. However, the diradical character in the ground state has been ruled out in the case of $[1,1,1]$ propellane by $\mathrm{Wu}$ et al., ${ }^{54}$ on the basis of a large vertical singlet-triplet gap (over 100 $\mathrm{kcal} / \mathrm{mol}$ ). The authors used a detailed Valence Bond analysis to classify the central C-C interaction as charge-shift bond. Lobayan et. al. ${ }^{55}$ also analyzed the density of unpaired electrons and its topology for this species at the CISD level of theory and ruled out the presence of a 3c-2e bond. Yet, the overall number of unpaired electrons $\left(N_{D}\right)$ they obtained at the CISD/6-31G* level of theory was quite significant (ca. 1.22).

We have performed the local spin analysis for [1,1,1]propellane and [2,2,2]propellane species at the CASSCF $(10,10) / c c-p V T Z / / U B 3 L Y P / c c-p V T Z$ level of theory. The results are gathered on Table 3. For [1,1,1]propellane, the central C-C distance is 1.568 , very similar to that of ethane for the same level of theory (1.528 ). We have found that the local spin on the central $\mathrm{C}$ atoms is completely negligible. In fact it is even smaller than the local spin con the $\mathrm{C}$ atoms of ethane described at the same level of theory ( 0.009 and 0.018 , respectively). Accordingly, the $\Delta^{(0)}$ value is very close to zero $(0.018)$, as expected for a nonradical species. For this level of theory we obtain an overall $N_{D}=0.46$, a value significantly smaller than that obtained by Lobayan and in more agreement with a nonradical picture. 
Table 3: Local spin on central $\mathrm{C}$ atoms, central $\mathrm{C}-\mathrm{C}$ bond orders (see text) and total number of unpaired electrons for ethane and several propellane species at the CASSCF $(10,10) / c c-p V T Z / / U B 3 L Y P / c c-p V T Z$ level of theory

\begin{tabular}{cccccc} 
Molecule & $\begin{array}{c}\mathrm{R}_{\mathrm{C}-\mathrm{C}} \\
(\AA)\end{array}$ & $\begin{array}{c}\mathrm{BO}_{\mathrm{C}-\mathrm{C}} \\
\text { (fluct.) }\end{array}$ & $\begin{array}{c}\mathrm{BO}_{\mathrm{C}-\mathrm{C}} \\
\text { (exch.) }\end{array}$ & $\left\langle\hat{S}^{2}\right\rangle_{A}$ & $N_{D}$ \\
\hline ethane & 1.528 & 0.78 & 0.91 & 0.018 & 0.318 \\
{$[1,1,1]$ propellane } & 1.568 & 0.44 & 0.66 & 0.009 & 0.463 \\
{$[2,2,2]$ propellane } & 1.536 & 0.77 & 0.87 & 0.028 & 0.095 \\
& $1.988^{a}$ & 0.33 & 0.43 & 0.313 & 1.082 \\
& 2.532 & 0.00 & 0.15 & 0.091 & 0.700 \\
\hline
\end{tabular}

${ }^{a}$ Transition state structure.

The potential energy surface of [2,2,2]propellane was studied in detail by Davidson $^{56}$ with different levels of theory with the $6-31 G^{*}$ basis set. There are two similar minimum energy structures for the singlet state. In the most strained one, the central $\mathrm{C}$-C distance is ca. $1.54 \AA$. Another minimum energy structure is found at a much longer $\mathrm{C}-\mathrm{C}$ distance $(2.54 \AA$ ) . Both are connected by a transition state structure at an intermediate distance of ca $2 \AA$. Both UB3LYP and CASSCF $(n, n)$ methods with $\mathrm{n}=2,4,8$ yield similar structures and energetics. The strained minimum structure is about 5-10 kcal/mol higher in energy than the stretched one, and the barrier for the interconversion (from the strained structure) is about $15-20 \mathrm{kcal} / \mathrm{mol} .{ }^{56}$ The multireference average quadratic coupled cluster (MRAQCC) results obtained by Antol et. al. ${ }^{57}$ with the same basis set were very similar to those reported by Davidson. It is worth to note that Davidson found that a low-energy broken-symmetry solution occurs from a C-C distance of ca. $1.7 \AA$ at the UHF/6-31G* level, whereas for UB3LYP the broken-symmetry solution only exists between C-C distances of 1.9 to $2.3 \AA$.

We have optimized the three structures at the UB3LYP/cc-pVTZ level of theory. Only the transition state structure lead to a broken-symmetry solution. Then we carried out single-point energy calculations at the CASSCF $(10,10) / \mathrm{cc}-\mathrm{pVTZ}$ level to perform the local spin analysis. The $a_{1}{ }^{\prime}, a_{2}$ " and two sets of $e$ ' and $e$ " orbitals were included in the active space. For the strained minimum $\left(\mathrm{R}_{\mathrm{C}-\mathrm{C}}=1.536 \AA\right)$ the local spin in the central $\mathrm{C}$ atoms is again negligible (0.028), and so is the number 
of unpaired electrons ( $N_{D}=0.095$ ). The corresponding $\Delta^{(0)}$ value is similar to that obtained for $[1,1,1]$ propellane.

In the stretched global minimum structure $\left(\mathrm{R}_{\mathrm{C}-\mathrm{C}}=2.532 \AA\right)$ there is no central $\mathrm{C}-\mathrm{C}$ bond. The bond orders are 0.15 and 0.00 for the exchange and fluctuation formulations, respectively). ${ }^{58}$ Also, the number of unpaired electrons is significantly larger than for the strained structure $\left(N_{D}=0.70\right)$. Yet, the local spin on the central $\mathrm{C}$ atoms is still very small (0.091). The $\Delta^{(2)}$ value of 0.33 is too large to consider this species as a diradical at all, specially when compared with the value for $\Delta^{(0)}$ (0.05). Since the UB3LYP description of this species is spin-restricted, $\Delta^{(0)}=0$ by definition at this level of theory.

The transition state structure $\left(\mathrm{R}_{\mathrm{C}-\mathrm{C}}=1.988 \AA\right)$ does exhibit significant local spin in the central $\mathrm{C}$ atoms (0.31), as well as larger number of effectively unpaired electrons $\left(N_{D}=1.08\right)$. The $\Delta^{(0)}$ and $\Delta^{(2)}$ values are 0.16 and 0.22 , respectively. Thus, the diradicaloid character at the transition state is larger than that of the minimum energy structures, but the species is still best pictured as a nonradical.

\section{Conclusions}

The general quantification of diradical character from wave function analysis is shown to be a non-trivial task, particularly for singlet states. In this work we illustrate how the descriptors obtained from a local spin analysis can be used to define a general measure of the diradical character. Indices $\Delta^{(0)}$ and $\Delta^{(2)}$ quantify deviation from a nonradical and a perfect diradical picture, respectively. The method reproduces the expected trend ortho-benzyne $<$ meta-benzyne $<$ para-benzyne of diradical character, for both the singlet and the triplet states. Also, it is found that diphospadiboretane and its diaza-analogue are best described as closed-shell and delocalized-spin species in their singlet and triplet states, respectively. The analysis performed on strained propellanes also confirm their nonradical nature, even in the absence of the central C-C bond. 


\section{Acknowledgments}

Financial help has been furnished by the Spanish MICINN Projects No. CTQ201123441/BQU. Financial support from MICINN and the FEDER fund (European Fund for Regional Development) was also provided by grant UNGI08-4E-003. Financial support from the Generalitat de Catalunya (SGR528 and Xarxa de Referència en Química Teòrica i Computacional) is also acknowledged. E.R-C. acknowledges support from the Spanish FPU program (Grant No. AP2008-01231) and from the EU under a Marie Curie Career Integration grant (PCI09-GA-2011-294240).

\section{References}

[1] L. Salem, Angew. Chem. Int. Ed. Engl., 1972, 11, 92.

[2] A. Rajca, Chem. Rev., 1994, 94, 871-893.

[3] Y. Jung and M. Head-Gordon, Chem. Phys. Chem., 2003, 4, 522-525.

[4] L. Noodleman, J. Chem. Phys., 1981, 74, 5737.

[5] L. Noodleman and E. R. Davidson, J. Chem. Phys., 1986, 109, 131.

[6] L. Salem and C. Rowland, Angew. Chem., 1972, 84, 86.

[7] V. Bachler, G. Olbrich, F. Neese and K. Wieghardt, Inorg. Chem., 2002, 41, 4179-4193.

[8] M. Seierstad, C. R. Kinsinger and C. J. Cramer, Angew. Chem. Int. Ed. Engl., 2002, 20, 41.

[9] X. Lopez, F. Ruipérez, M. Piris, J. M. Matxain and J. M. Ugalde, Comp. Phys. Com., 2011, 12, 1061-1065.

[10] P. Rivero, C. A. Jiménez-Hoyos and G. E. Scuseria, J. Phys. Chem. B, 2013, 117, 12750-12758.

[11] K. Kamada, K. Ohta, A. Shimizu, T. Kubo, R. Kishi, H. Takahashi, E. Botek, B. Champagne and M. Nakano, J. Phys. Chem. Lett., 2010, 1, 937-940.

[12] D. Herebian, K. E. Wieghardt and F. Neese, J. Am. Chem. Soc., 2003, 125, 10997-11005. 
[13] F. Plasser, H. Pašalić, M. H. Gerzabek, F. Libisch, R. Reiter, J. Burgdörfer, T. Müller, R. Shepard and H. Lischka, Angew. Chem. Int. Ed. Engl., 2013, 52, 2581-2584.

[14] K. Takatsuka, T. Fueno and K. Yamaguchi, Theor. Chim. Acta (Berlin), 1978, 48, 175-183.

[15] V. N. Staroverov and E. R. Davidson, J. Am. Chem. Soc., 2000, 122, 186-187.

[16] V. N. Staroverov and E. R. Davidson, J. Am. Chem. Soc., 2000, 122, $7377-$ 7385 .

[17] M.-J. Cheng and C.-H. Hu, Mol. Phys., 2003, 101, 1319-1323.

[18] L. Lain, A. Torre, R. C. Bochicchio and R. Ponec, Chem. Phys. Lett., 2001, 346, 283-287.

[19] I. Mayer, Int. J. Quant. Chem., 1986, 29, 73-84.

[20] I. Mayer, Int. J. Quant. Chem., 1986, 29, 477-483.

[21] A. E. Clark and E. R. Davidson, J. Chem. Phys., 2001, 115, 7382-7392.

[22] M. Reiher, Faraday Discuss., 2006, 135, 97-124.

[23] I. Mayer, Chem. Phys. Lett., 2007, 440, 357-359.

[24] I. Mayer and E. Matito, Phys. Chem. Chem. Phys., 2010, 12, 11308-11314.

[25] D. R. Alcoba, A. Torre, L. Lain and R. C. Bochicchio, J. Chem. Theory Comput., 2011, 7, 3560-3566.

[26] E. Ramos-Cordoba, E. Matito, P. Salvador and I. Mayer, Phys. Chem. Chem. Phys., 2012, 14, 15291-15298.

[27] E. Ramos-Cordoba, E. Matito, I. Mayer and P. Salvador, J. Chem. Theory Comput., 2012, 8, 1270-1279.

[28] C. Herrmann, L. Yu and M. Reiher, J. Comput. Chem., 2006, 27, 1223-1239.

[29] C. Herrmann, M. Reiher and B. A. Hess, J. Chem. Phys., 2005, 122, 034102.

[30] E. Ramos-Cordoba, P. Salvador and M. Reiher, Chem. Eur. J., 2013, 19, $15267-15275$.

[31] E. Ramos-Cordoba and P. Salvador, J. Chem. Theory Comput., 2014, DOI: 10.1021/ct401009p. 
[32] A. E. Clark and E. R. Davidson, J. Phys. Chem. A, 2002, 106, 6890-6896.

[33] A. E. Clark and E. R. Davidson, Mol. Phys., 2002, 100, 373-383.

[34] A. Clark, E. Davidson and J. Zaleski, J. Am. Chem. Soc., 2001, 123, 26502657.

[35] A. Clark and E. Davidson, Phys. Chem. Chem. Phys., 2007, 9, 1881-1894.

[36] J. Oliva, D. Alcoba, L. Lain and A. Torre, Theor. Chem. Acc., 2013, 132, 1-6.

[37] M. J. Frisch, G. W. Trucks, H. B. Schlegel, G. E. Scuseria, M. A. Robb, J. R. Cheeseman, J. A. Montgomery, Jr., T. Vreven, K. N. Kudin, J. C. Burant, J. M. Millam, S. S. Iyengar, J. Tomasi, V. Barone, B. Mennucci, M. Cossi, G. Scalmani, N. Rega, G. A. Petersson, H. Nakatsuji, M. Hada, M. Ehara, K. Toyota, R. Fukuda, J. Hasegawa, M. Ishida, T. Nakajima, Y. Honda, O. Kitao, H. Nakai, M. Klene, X. Li, J. E. Knox, H. P. Hratchian, J. B. Cross, V. Bakken, C. Adamo, J. Jaramillo, R. Gomperts, R. E. Stratmann, O. Yazyev, A. J. Austin, R. Cammi, C. Pomelli, J. W. Ochterski, P. Y. Ayala, K. Morokuma, G. A. Voth, P. Salvador, J. J. Dannenberg, V. G. Zakrzewski, S. Dapprich, A. D. Daniels, M. C. Strain, O. Farkas, D. K. Malick, A. D. Rabuck, K. Raghavachari, J. B. Foresman, J. V. Ortiz, Q. Cui, A. G. Baboul, S. Clifford, J. Cioslowski, B. B. Stefanov, G. Liu, A. Liashenko, P. Piskorz, I. Komaromi, R. L. Martin, D. J. Fox, T. Keith, M. A. Al-Laham, C. Y. Peng, A. Nanayakkara, M. Challacombe, P. M. W. Gill, B. Johnson, W. Chen, M. W. Wong, C. Gonzalez and J. A. Pople, Gaussian 03, Revision C.02, Gaussian, Inc., Pittsburgh, PA, 2003.

[38] E. Matito and F. Feixas, DMN program, 2009, University of Girona (Spain) and University of Szczecin (Poland).

[39] P. Salvador and E. Ramos-Cordoba, APOST-3D program, 2012, Universitat de Girona (Spain).

[40] P. Salvador and E. Ramos-Cordoba, J. Chem. Phys., 2013, 139, 071103.

[41] E. Matito, M. Solà, P. Salvador and M. Duran, Faraday Discuss., 2007, 135, 325-345.

[42] V. N. Staroverov and E. R. Davidson, Chem. Phys. Lett., 2000, 330, 161-168.

[43] M. Head-Gordon, Chem. Phys. Lett., 2003, 372, 508-511.

[44] R. C. Bochicchio, A. Torre and L. Lain, Chem. Phys. Lett., 2003, 380, 486487. 
[45] M. Head-Gordon, Chem. Phys. Lett., 2003, 488-489.

[46] P. G. Wenthold, R. R. Squires and W. Lineberger, J. Am. Chem. Soc., 1998, 120, 5279-5290.

[47] F. De Proft, P. von Ragué Schleyer, J. H. van Lenthe, F. Stahl and P. Geerlings, Chem. Eur. J., 2002, 8, 3402-3410.

[48] E. B. Wang, C. A. Parish and H. Lischka, J. Chem. Phys., 2008, 129, 044306.

[49] A. Clark and E. Davidson, J. Am. Chem. Soc., 2001, 123, 10691-10698.

[50] P. Nachtigall and K. D. Jordan, J. Am. Chem. Soc., 1992, 114, 4743-4747.

[51] M. Filatov and S. Shaik, J. Phys. Chem. A, 1999, 103, 8885-8889.

[52] D. Scheschkewitz, H. Amii, H. Gornitzka, W. Schoeller, D. Bourissou and G. Bertrand, Science, 2002, 295, 1880-1881.

[53] Y. Jung and M. Head-Gordon, J. Phys. Chem. A, 2003, 107, 7475-7481.

[54] W. Wu, J. Gu, J. Song, S. Shaik and P. C. Hiberty, Angew. Chem. Int. Ed. Engl., 2009, 48, 1407-1410.

[55] R. Lobayan and R. Bochicchio, Chem. Phys. Lett., 2013, 557, 154-158.

[56] E. Davidson, Chem. Phys. Lett., 1998, 284, 301-307.

[57] I. Antol, M. Eckert-Maksić, H. Lischka and Z. B. Maksić, Eur. J. Org. Chem., 2007, 2007, 3173-3178.

[58] J. G. Ángyán, E. Rosta and P. R. Surján, Chem. Phys. Lett., 1999, 299, 1-8. 


\title{
SUPPLEMENTARY INFORMATION
}

\section{Diradical character from the local spin analysis}

\author{
Eloy Ramos-Cordoba, Pedro Salvador \\ Institut de Química Computacional i Catàlisi (IQCC) and Departament de Química, \\ University of Girona, 17071 Girona, Spain \\ e-mail: Pedro.Salvador@udg.edu
}

\section{The local spin analysis}

In the local spin analysis, the $\left\langle\hat{S}^{2}\right\rangle_{A}$ values indicate and quantify the presence of local spin within the molecule, namely on atom/fragment $A$. The magnitude and sign of the diatomic contributions $\left\langle\hat{S}^{2}\right\rangle_{A B}$ with $B \neq A$ inform about the nature of the couplings between these local spins. The physical interpretation of the $\left\langle\hat{S}^{2}\right\rangle_{A}$ and particularly $\left\langle\hat{S}^{2}\right\rangle_{A B}$ values is somewhat intricate, and has been recently discussed in detail in several papers. ${ }^{1,2}$ We provide here a brief account of its general characteristics for ideal systems.

When two perfectly localized spins are coupled as a singlet, a proper multireference wave function is needed to account for the spin properties of the system. In that case, the local spin analysis yields $\left\langle\hat{S}^{2}\right\rangle_{A}=3 / 4$ and $\left\langle\hat{S}^{2}\right\rangle_{A B}=-3 / 4$, which account for the expected overall $\left\langle\hat{S}^{2}\right\rangle=0$. The $\left\langle\hat{S}^{2}\right\rangle_{A}$ value is consistent with the corresponding $\left\langle\hat{S}^{2}\right\rangle=s(s+1)$ value for the isolated one-electron system and the negative sign of $\left\langle\hat{S}^{2}\right\rangle_{A B}$ indicates that the two local spins are coupled as a singlet (entangled). With a single-determinant broken symmetry description of the same system one would obtain similarly $\left\langle\hat{S}^{2}\right\rangle_{A}=3 / 4$, but now $\left\langle\hat{S}^{2}\right\rangle_{A B}=-1 / 4$, for an overall value of $\left\langle\hat{S}^{2}\right\rangle=1$. Both $\left\langle\hat{S}^{2}\right\rangle_{A}$ and $\left\langle\hat{S}^{2}\right\rangle_{A B}$ monotonically decrease as the two spins become more amd more delocalized. In the limiting case of a closedshell single-determinant description, all local spin contributions exactly vanish. 
If the two perfectly localized spins on centers $A$ and $B$ are parallel, the local spin analysis would yield $\left\langle\hat{S}^{2}\right\rangle_{A}=3 / 4$ and $\left\langle\hat{S}^{2}\right\rangle_{A B}=1 / 4$, for an overall value of $\left\langle\hat{S}^{2}\right\rangle=$ 2 , as expected for a triplet. The one-center term is again consistent with a oneelectron system, and the positive sign of the diatomic contribution now indicates that the local spins are parallel. In a parallel-spins situation, if the two spins are not perfectly localized the value of $\left\langle\hat{S}^{2}\right\rangle_{A B}$ decreases, but that of $\left\langle\hat{S}^{2}\right\rangle_{A}$ increases. The latter is an indication of partial triplet character on the given center.

\section{Local spin analysis of diradicals and diradicaloids}

Tetramethyleneethane (TME) is a well-studied diradical. ${ }^{3,4}$ This molecule has been optimized under three different symmetry constrains, namely $D_{2}, D_{2 h}$ and $D_{2 d}$ at the CASSCF level of theory. The active space included 6 electrons and 6 orbitals (in the $D_{2 h}$ symmetry they correspond to the set of $6 \pi$ orbitals.) The lowest energy structure has $D_{2}$ symmetry, with a $\mathrm{C}_{1}-\mathrm{C}_{2}-\mathrm{C}_{4}-\mathrm{C}_{5}$ dihedral angle $(\alpha)$ of $70.6 \mathrm{deg}$ (see Figure 1).

The results of the local spin analysis are gathered on Table 1. The atomic and diatomic spin components are almost independent of the rotation with respect to the central C-C bond. The vertical singlet-triplet gaps, sometimes used to assess the radical character, ${ }^{4}$ are not too different for the $D_{2}, D_{2 h}$, and $D_{2 d}$ structures (-1.33, 3.91 , and $-2.05 \mathrm{kcal} / \mathrm{mol}$, respectively). We will focus on the results obtained for the global minimum ( $D_{2}$ symmetry). The main spin centers are $\mathrm{C}_{1}$ and the symmetry equivalent $\mathrm{C}_{3}, \mathrm{C}_{5}$, and $\mathrm{C}_{6}$, with $\left\langle\hat{S}^{2}\right\rangle_{\mathrm{C}}$ values of 0.35 and 0.36 for the singlet and triplet states (the local spin involving the $\mathrm{H}$ atoms is negligible). The diatomic spin terms $\left\langle\hat{S}^{2}\right\rangle_{\mathrm{C}_{1}, \mathrm{C}_{2}}$ and $\left\langle\hat{S}^{2}\right\rangle_{\mathrm{C}_{1}, \mathrm{C}_{3}}$ (and their symmetry equivalents) also equal in both electronic states. The sign of these spin contributions indicates the alternation of the spins within each allyl fragment, as indicated in Figure 1. The main differences between the local spin distribution of the singlet and triplet states are found in the diatomic terms involving the $\mathrm{C}$ atoms on the different allyl fragments. In the singlet state, the atoms 1,3 , and 4 have parallel spins, as indicated by the sign of $\left\langle\hat{S}^{2}\right\rangle_{\mathrm{C}_{1}, \mathrm{C}_{3}}$ and $\left\langle\hat{S}^{2}\right\rangle_{\mathrm{C}_{1}, C_{4}}$ terms, whereas centers 2,5 , and 6 exhibit antiparallel arrangement with respect to them. 


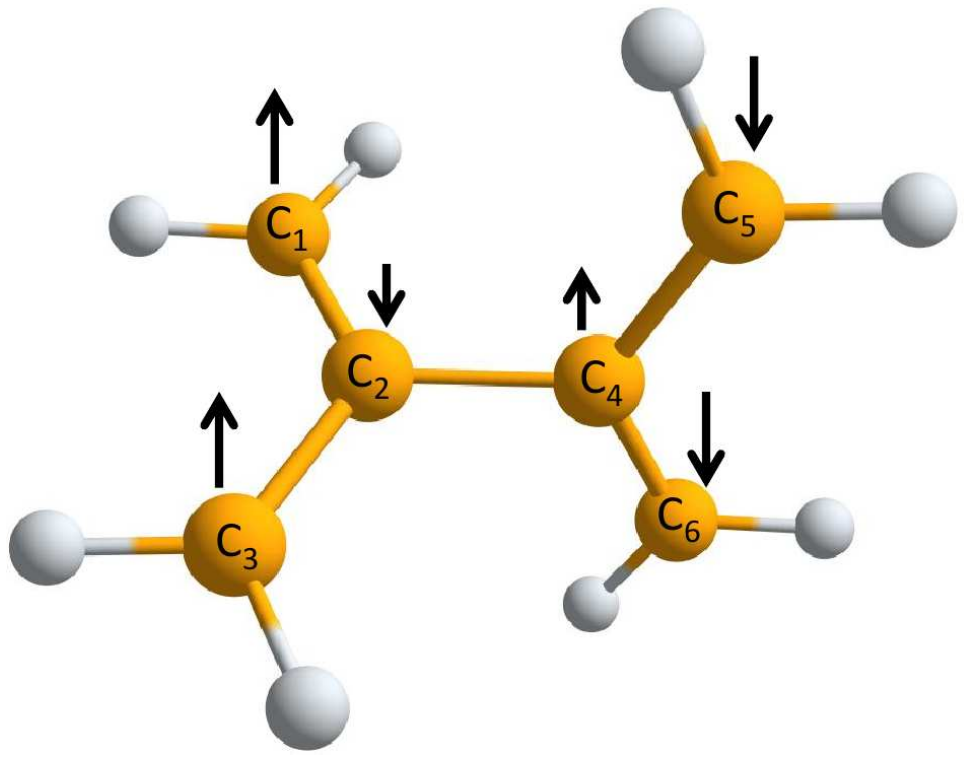

Figure 1: Numbering scheme of tetrathyleneethane (TME) and local spin distribution of the singlet state.

The diatomic terms $\left\langle\hat{S}^{2}\right\rangle_{\mathrm{C}_{1}, \mathrm{C}_{5}}$ present a rather small value of -0.17 . However, this value accounts for most of the expected diatomic spin contribution of $-3 / 4$ between the two allyl centers, as there are four such diatomic contributions equivalent by symmetry. In the triplet state, the local spins on the $\mathrm{C}$ atoms of one of the allyl moieties are flipped with respect to the singlet state. The four symmetry-equivalent diatomic terms $\left\langle\hat{S}^{2}\right\rangle_{\mathrm{C}_{1}, \mathrm{C}_{5}}=0.05$ account for the spin-spin interactions between the allyl moieties.

The diatomic spin contribution between atoms $\mathrm{C}_{2}$ and $\mathrm{C}_{4}$ is almost zero. Moreover, the one- and two-center contributions involving atoms $\mathrm{C}_{1}, \mathrm{C}_{2}$ and $\mathrm{C}_{3}$ are very similar to those observed for a single allyl radical. ${ }^{5}$ In fact, summing up all oneand two-center contributions for all atoms of each allyl moiety gives a local spin on each fragment very close to $3 / 4$ in all cases (see bottom of Table 1). Thus, the TME molecule can be regarded as a diradical made up from two independent allyl radicals bonded by the central carbon atoms. The local spin analysis unravels the spin distribution among all centers or fragments on the same footing both different electronic states, i.e., even if no spin density exists. 
Table 1: Local spin analysis of the TME molecule for the singlet (S) and triplet (T) states of different geometries.

\begin{tabular}{ccccccc}
\hline Atom/Atom pair & \multicolumn{2}{c}{$D_{2}(\alpha=70.6)$} & \multicolumn{2}{c}{$D_{2 h}(\alpha=0)$} & \multicolumn{2}{c}{$D_{2 d}(\alpha=90)$} \\
Fragment/ Fragment pair & $\mathrm{S}$ & $\mathrm{T}$ & $\mathrm{S}$ & $\mathrm{T}$ & $\mathrm{S}$ & $\mathrm{T}$ \\
\hline $\mathrm{C}_{1}$ & 0.36 & 0.37 & 0.36 & 0.37 & 0.35 & 0.37 \\
$\mathrm{C}_{2}$ & 0.14 & 0.14 & 0.14 & 0.14 & 0.14 & 0.14 \\
$\mathrm{C}_{1}, \mathrm{C}_{2}$ & -0.09 & -0.09 & -0.08 & -0.09 & -0.09 & -0.09 \\
$\mathrm{C}_{1}, \mathrm{C}_{3}$ & 0.08 & 0.08 & 0.08 & 0.08 & 0.08 & 0.08 \\
$\mathrm{C}_{1}, \mathrm{C}_{4}$ & 0.03 & -0.01 & 0.03 & 0.00 & 0.03 & -0.01 \\
$\mathrm{C}_{1}, \mathrm{C}_{5}$ & -0.17 & 0.05 & -0.17 & 0.04 & -0.17 & 0.05 \\
$\mathrm{C}_{1}, \mathrm{C}_{6}$ & -0.18 & 0.05 & -0.19 & 0.04 & -0.17 & 0.05 \\
$\mathrm{C}_{2}, \mathrm{C}_{4}$ & -0.01 & -0.01 & -0.02 & -0.02 & -0.01 & 0.00 \\
allyl & 0.77 & 0.80 & 0.79 & 0.81 & 0.76 & 0.79 \\
allyl $_{1}$, allyl & -0.77 & 0.20 & -0.79 & 0.19 & -0.76 & 0.21 \\
\hline
\end{tabular}

Diphosphadiboretanes and their analogues are some of the most controversial diradicaloid systems discussed in the literature. For these systems we have carried out the local spin analysis with both an unrestricted single-determinant (UB3LYP) and a $\operatorname{CASSCF}(2,2)$ wave function for comparision, and for both their singlet and triplet states. The results are gathered on Table 2.

Table 2: Local spin analysis of four-member ring diradicaloids for different spin states and levels of theory

\begin{tabular}{lccccc}
\hline \multirow{2}{*}{ Molecule } & & \multicolumn{4}{c}{$\left\langle\hat{S}^{2}\right\rangle_{A} /\left\langle\hat{S}^{2}\right\rangle_{A B}$} \\
& Atom/Atom pair & \multicolumn{2}{c}{ CASSCF } & \multicolumn{2}{c}{ UB3LYP } \\
& & Singlet & Triplet & Singlet & Triplet \\
\hline \multirow{2}{*}{$(\mathrm{CH})_{2}\left(\mathrm{C}^{\prime} \mathrm{H}_{2}\right)_{2}$} & C & 0.58 & 0.59 & 0.59 & 0.59 \\
& C-C & 0.05 & 0.05 & 0.05 & 0.06 \\
& B & -0.46 & 0.15 & -0.16 & 0.16 \\
$(\mathrm{BH})_{2}\left(\mathrm{PH}_{2}\right)_{2}$ & P & 0.10 & 0.27 & 0.00 & 0.32 \\
& B-B & -0.04 & 0.26 & 0.00 & 0.22 \\
& B & 0.19 & 0.28 & 0.00 & 0.05 \\
$(\mathrm{BH})_{2}\left(\mathrm{NH}_{2}\right)_{2}$ & N & 0.14 & 0.25 & 0.15 & 0.31 \\
& B-B & -0.07 & 0.03 & -0.03 & 0.05 \\
\hline
\end{tabular}


The local spin values (atomic terms) for singlet states are very similar for CASSCF $(2,2)$ and UB3LYP methods, provided a broken-symmetry solution is found for the latter. For singlet states, the species with a larger local spin contributions is $(\mathrm{CH})_{2}\left(\mathrm{CH}_{2}\right)_{2}$. The value for the $\operatorname{CASSCF}(2,2)$ wave function $(0.58)$ is not too far from that expected for a perfectly localized electron (3/4). In $(\mathrm{BH})_{2}\left(\mathrm{NH}_{2}\right)_{2}$ the local spin is significantly smaller (0.19), which should indicate a much weaker diradical character. In the case of the diphosphadiboretane species the local spin is almost negligible (0.10), consistent with a residual diradical character.

For triplet states the local spin analysis yields very similar one- and two-center contributions for both methods. This is not surprising since with a $\operatorname{CASSCF}(2,2)$ approach the $m_{S}=|S|$ state is described by a ROHF wave function. The atomic contributions of $(\mathrm{CH})_{2}\left(\mathrm{CH}_{2}\right)_{2}$ are essentially the same as in the singlet state. The diatomic term involving the two main local spin centers is now positive, indicating parallel arrangement of the spins. In the case of $(\mathrm{BH})_{2}\left(\mathrm{PH}_{2}\right)_{2}$ and $(\mathrm{BH})_{2}\left(\mathrm{NH}_{2}\right)_{2}$ the four atoms of the ring exhibit similar but small contributions. In fact, the sum of the terms reported on Table 2 is still far from the overall $\left\langle\hat{S}^{2}\right\rangle \approx 2$ value. This is because the hydrogen atoms (omitted thus far) exhibit small but significant contributions of

ca. 0.05-0.10. Thus, these species do not exhibit significant spin centers and the molecular spin is delocalized over all atoms.

\section{References}

[1] E. Ramos-Cordoba, P. Salvador and M. Reiher, Chem. Eur. J., 2013, 19, 1526715275 .

[2] E. Ramos-Cordoba and P. Salvador, J. Chem. Theory Comput., 2014, DOI: $10.1021 / \mathrm{ct} 401009 \mathrm{p}$.

[3] P. Nachtigall and K. D. Jordan, J. Am. Chem. Soc., 1992, 114, 4743-4747.

[4] M. Filatov and S. Shaik, J. Phys. Chem. A, 1999, 103, 8885-8889.

[5] E. Ramos-Cordoba, E. Matito, P. Salvador and I. Mayer, Phys. Chem. Chem. Phys., 2012, 14, 15291-15298. 